\title{
Muon Spin Rotation Studies of Spin Dynamics at Avoided Level Crossings in $\operatorname{LiY}_{0.998} \mathrm{Ho}_{0.002} \mathrm{~F}_{4}$
}

\author{
M. J. Graf, ${ }^{1}$ J. Lago, ${ }^{2}$ A. Lascialfari, ${ }^{2,3}$ A. Amato, ${ }^{4}$ C. Baines, ${ }^{4}$ S. R. Giblin, ${ }^{5}$ J. S. Lord, ${ }^{5}$ A. M. Tkachuk, ${ }^{6}$ and B. Barbara ${ }^{7}$ \\ ${ }^{1}$ Department of Physics, Boston College, Chestnut Hill, Massachusetts 02467, USA \\ ${ }^{2}$ Department of Physics "A. Volta”, University of Pavia, and CNR-INFM, I27100 Pavia, Italy \\ ${ }^{3}$ Inst. of General Physiology and Biological Chemistry, Univ. of Milano, I20134 Milano, Italy \\ and S3-CNR-INFM, I41100 Modena, Italy \\ ${ }^{4}$ Paul Scherrer Institute, CH 5232 Villigen PSI, Switzerland \\ ${ }^{5}$ Rutherford Appleton Laboratory, Chilton Didcot, Oxfordshire OX11 OQX, United Kingdom \\ ${ }^{6}$ St. Petersburg State University of Information Technology, Mechanics and Optics, 199034, St. Petersburg, Russia \\ ${ }^{7}$ Institut Néel, Département Nanosciences, CNRS, 38042 Grenoble Cedex-09, France
}

(Received 5 September 2007; published 27 December 2007)

\begin{abstract}
We have studied the $\mathrm{Ho}^{3+}$ spin dynamics for $\mathrm{LiY}_{0.998} \mathrm{Ho}_{0.002} \mathrm{~F}_{4}$ via the positive muon $\left(\mu^{+}\right)$transverse field depolarization rate $\lambda_{\mathrm{TF}}$ as a function of temperature and magnetic field. We find sharp minima in $\lambda_{\mathrm{TF}}(H)$ at fields for which the $\mathrm{Ho}^{3+}$ ion system has field-induced (avoided) level crossings. The reduction scales with calculated level repulsions, suggesting that $\mu^{+}$depolarization by slow fluctuations of nonresonant $\mathrm{Ho}^{3+}$ spin states is partly suppressed when resonant tunneling opens new fluctuation channels at frequencies much greater than the muon precession frequency.
\end{abstract}

DOI: $10.1103 /$ PhysRevLett.99.267203

Zero-dimensional magnetic systems exhibit spin dynamics which are dominated by quantum effects at low temperatures, as made evident by recent studies of singlemolecule magnets (SMM) [1]. In SMM, a cluster of transition metal ions is imbedded within a large organic molecule, with the ions becoming locked into either nonzero (e.g., $\mathrm{Mn} 12$ or $\mathrm{Fe} 8$ ) or zero-spin states (e.g., Cr8) when the thermal energy is much smaller than the exchange coupling. The easy-axis anisotropy produces a large energy barrier (typically several tens of degrees K) to spin reversal, which can be overcome by thermal activation, quantum tunneling, or a combination of both mechanisms. Application of a magnetic field can produce level-crossings through Zeeman splitting of the low-lying energy states, and intrinsic (e.g., transverse crystal-field and hyperfine fields) or extrinsic (applied transverse fields) state mixing produces tunnel splittings and avoided level crossings (ALCs). The enhanced low-temperature spin transition rates at ALCs produce steps in the sample magnetization curves, as first observed in Mn12 [2].

NMR is an effective probe for studying the spin dynamics in these systems in moderate-to-strong magnetic fields [1]. At low temperatures in selected high-symmetry systems (e.g., molecular rings), the enhanced transition rate at ALCs results in peaks in the magnetic field dependence of the spin-lattice relaxation rate $1 / T_{1}$. Positive muon spin rotation/relaxation $\left(\mu^{+} \mathrm{SR}\right)$ should be an important complementary probe to the NMR measurements on SMM, as this highly sensitive local probe can be used from zero to high magnetic fields, and the local field can be probed at locations other than the active nuclear site. In fact, we know of no example where measurements have exhibited an enhanced spin transition rates at ALCs in SMM systems [3], which in part can be attributed to the difficulty in producing large single crystals.
PACS numbers: 75.45.+j, 76.30.Kg, 76.60.Es, 76.75.+i

The single-ion magnet $\mathrm{LiY}_{1-x} \mathrm{Ho}_{x} \mathrm{~F}_{4}$ exhibits many of the same properties as SMMs. The $J=8$ electronic levels of the $\mathrm{Ho}^{3+}$ ion are split by the crystal electric field producing a low-lying ground state doublet, which itself is split by hyperfine coupling with the ${ }^{165}$ Ho nucleus $(I=$ $7 / 2$ ). An external magnetic field component along the quantization axis ( $c$-axis in this Scheelite structure) produces ALCs for $H_{n}=23 n \mathrm{mT}$, with $-7 \leq n \leq 7$ and $n$ being an integer (see Fig. 1). Thus, for small $x$, where the Ho-Ho dipolar coupling is weak, the spin dynamics are similar to those for the SMMs, e.g., magnetization measurements show steps at the ALCs at low temperatures [4]. Recent ${ }^{19} \mathrm{~F}$ NMR low-temperature measurements of the magnetic field dependence of $1 / T_{1}$ exhibit strikingly sharp peaks at ALCs for $n=4-7$ [5].

Calculations [4] of the variation of the single-ion electronuclear energy levels with the component of the magnetic field along the quantization axis also predict the degree of state mixing at ALCs, neglecting crystalline

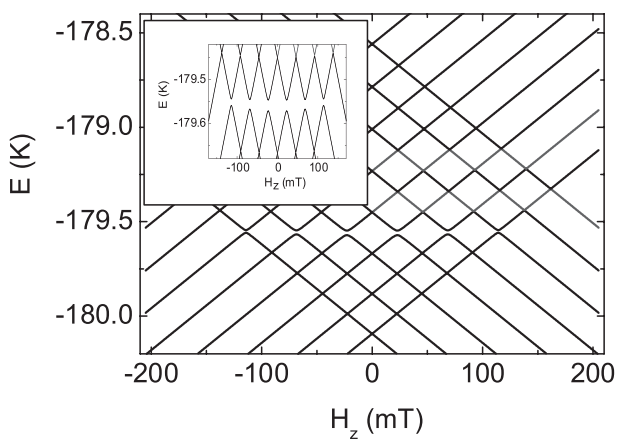

FIG. 1. Calculated magnetic field variation of the hyperfine split ground state electronic energy levels. Inset: closer view of the dominant tunnel splittings (adapted from Ref. [6]). 
defects or a component of the applied field perpendicular to the $c$-axis. The resultant tunnel splittings arise from the combined effect of the crystal field and the hyperfine interactions. The crystal field acting on the total angular momentum $\boldsymbol{J}$ gives a quasi-Ising ground state doublet $\Gamma_{3,4}$, with the next highest levels being singlets, resulting from the same $\Gamma_{2}$, at 10 and $36 \mathrm{~K}$ above the ground-state, and two nearly-degenerate $\Gamma_{1}$ states at $70 \mathrm{~K}$ above the ground state. The hyperfine interactions given by the Hamiltonian

$$
\mathcal{H}=A_{J}\left\{J_{z} I_{z}+(1 / 2)\left(J_{+} I_{-}+J_{-} I_{+}\right)\right\},
$$

where $A_{J}$ is the hyperfine constant and $J_{i}$ and $I_{i}$ are the electronic and nuclear angular momentum components, respectively, multiply the degeneracy of each state by 8 . First order perturbation calculations modify the energy by $A_{J} M m_{I}$, where $M$ and $m_{I}$ are the electronic and nuclear spin quantum numbers, and the $\Gamma_{3,4}$ doublet transforms into 8 magnetic field dependent levels of effective electronic spin $1 / 2$ and 8 levels with effective electronic spin $-1 / 2$. Including the off-diagonal part of Eq. (1), second order perturbation theory shows that the $\Gamma_{3,4}$ states mix with those of $\Gamma_{2}$ or $\Gamma_{1}$ when the selection rule $\Delta m_{I} / 2=k$, where $k$ is an odd integer, is fulfilled. The resultant repulsion at the $\Gamma_{3,4}$ crossings increases with the repulsion of singlets. Thus, the crossings at $n= \pm 1, \pm 3$, and \pm 5 and energy $-179.55 \mathrm{~K}$ have large tunnel splittings of 25,22 , and $16 \mathrm{mK}$, respectively, (mixing via $\Gamma_{2}$ ), while all other splittings are very small (Fig. 1). This energy level diagram was confirmed in detail by recent EPR measurements [6].

The $\mathrm{LiY}_{0.998} \mathrm{Ho}_{0.002} \mathrm{~F}_{4}$ system offers many distinct advantages over SMM for $\mu^{+}$SR studies, the most obvious being that large, high quality, and well-characterized single crystals can be studied. Earlier longitudinal field $\mu^{+}$SR measurements [7] showed a very weak increase in the muon depolarization rate at the $n=1 \mathrm{ALC}$, but the applied field greatly depressed the depolarization rate, ruling out any detailed quantitative studies. In this work, we show that transverse field $\mu^{+}$SR is a highly sensitive probe of the $\mathrm{Ho}^{3+}$ spin dynamics, and we find sharp decreases in the muon depolarization rate at fields corresponding to ALCs, with relative sizes in good agreement with the calculations described above.

A large single crystal with nominal Ho concentration $x=0.002$ was grown and cut into plates with dimensions $3 \times 8 \times 30 \mathrm{~mm}^{3}$, with the crystalline $c$-axis aligned along the $3 \mathrm{~mm}$ edge. We measured the ${ }^{19} \mathrm{~F} 1 / T_{1}$ as a function of magnetic field at $1.7 \mathrm{~K}$ and observed sharp peaks in $1 / T_{1}$ at ALCs with $n=5,6$, and 7 , as seen for a different crystal in Ref. [5].

In transverse field $\mu^{+} \mathrm{SR}$, a muon with spin aligned perpendicular to an applied magnetic field is implanted in a sample and precesses in the local magnetic field. At some later time, the muon decays, and the resulting positron is preferentially emitted along the muon spin polarization axis and detected. After several million decay events, one constructs a time histogram of the detector signal asymmetry $A(t)$ [8] which is proportional to the muon depolarization function $P_{\mu}(t)$, defined as the projection of the spin polarization along the initial polarization direction. A variation of the local magnetic field between muon stopping sites will produce a dephasing of the oscillatory $P_{\mu}(t)$ due to the spread of Larmor frequencies for the individual muon decay events, so the measurements are extremely sensitive to magnetic inhomogeneity. The $\mu^{+}$SR measurements were conducted utilizing the GPS and LTF spectrometers on the $\pi M 3$ continuous beam line at the Paul Scherrer Institute (PSI), spanning the range $30 \mathrm{mK} \leq T \leq 50 \mathrm{~K}$. The beam momentum and external magnetic field were aligned parallel to the crystalline $c$-axis. We utilized the spin-rotated mode, where the muon spin is aligned at approximately 50 degrees to the beam momentum. The transverse depolarization is then monitored with detectors oriented along a line perpendicular to the beam momentum.

Earlier zero field $\mu^{+}$SR results on these samples [7] showed that nearly all the muons are trapped by pairs of adjacent $\mathrm{F}^{-}$ions, forming the stable three-spin system F- $\mu$-F [9]. In zero-magnetic field, this system exhibits spin-flip oscillations at three nonzero frequencies $(3-$ $\sqrt{3}) \omega_{D} / 2, \sqrt{3} \omega_{D},(3+\sqrt{3}) \omega_{D} / 2$, where $f_{D}=\omega_{D} / 2 \pi$ is determined by the average separation between the muon and the $\mathrm{F}$ ions and their gyromagnetic ratios. The relative amplitudes of these components are determined by the orientation of the muon polarization relative to the line joining the adjoining $\mathrm{F}$ ions. Our new analysis indicates that (1) the most likely muon stopping sites are the four magnetically equivalent unit cell locations where $2 \mathrm{~F}$ ions are closest, and the F ions are pulled in from a separation of 0.260 to $0.239 \mathrm{~nm}$ by the muon; (2) below $10 \mathrm{~K}$, the damping of the oscillations increases significantly, and (3) the fitted value of $f_{D}$ increases slightly below $6 \mathrm{~K}$, from 0.211 to $0.220 \mathrm{MHz}$. The first result is consistent with results on the $\mathrm{F}-\mu-\mathrm{F}$ formation in comparable insulating fluorides. The second result is consistent with the transverse field results given below, which show the onset at low temperatures of quasistatic magnetic disorder associated with the $\mathrm{Ho}^{3+}$ ions. Monte Carlo calculations [10] for $\mathrm{F}-\mu$-F oscillations in the presence of a Gaussian distribution of static moments of width $\Delta$ show that the observed $f_{D}$ in zero field will have a small positive correction that increases as $\Delta^{2}$, providing a qualitative understanding of the third result.

We now describe the variation of the depolarization rate with temperature in a magnetic field of $23 \mathrm{mT}$, corresponding to the $n=1$ series of avoided level crossings (Fig. 1). The oscillatory asymmetry $A(t)$ exhibits a damping which can be well fit by a stretched exponential function so that $A(t)=A_{0} \exp \left[-\left(\lambda_{T F} t\right)^{\beta}\right\rfloor \cos \left(\omega_{L} t+\phi\right)$, where $\omega_{L}$ is the Larmor frequency. All $\chi^{2}$ values (per degree of freedom) lie in the range 0.9997-1.0313. In Fig. 2, we show the 


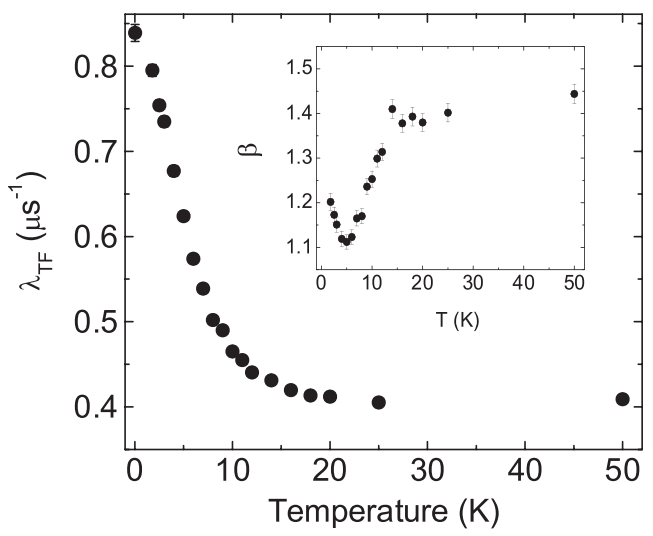

FIG. 2. The temperature variation of the depolarization rate at a fixed field of $23 \mathrm{mT}$. Inset: Temperature dependence of the exponent of the fitted stretched exponential function.

temperature dependence of the depolarization rate $\lambda_{\mathrm{TF}}(T)$; the inset shows the variation of the exponent $\beta$. At $T=$ $50 \mathrm{~K}, \lambda_{\mathrm{TF}}$ has a relatively large value of $0.409 \pm$ $0.003 \mu \mathrm{s}^{-1}$, reflecting the quasistatic magnetic disorder produced by the various magnetic nuclear species. As the temperature is lowered, it remains roughly constant until about $20 \mathrm{~K}$, below which it increases sharply, reaching a value of $\lambda_{\mathrm{TF}}=0.866 \pm 0.008 \mu \mathrm{s}^{-1}$ at $30 \mathrm{mK}$. The increase results from decoherence of the precessional motion of muon spins due to the increase of quasistatic disorder at temperatures lower than the barrier for $\mathrm{Ho}^{3+}$ spin reversal. The $\Gamma_{2}$ singlets, at roughly 10 and $36 \mathrm{~K}$ above the ground state, induce strong longitudinal and transverse fluctuations for $T>10 \mathrm{~K}$. These spin fluctuations are too fast to affect the local disorder on the muon precessional time scale. Cooling below the spin reversal barrier of $10 \mathrm{~K}$, the $\mathrm{Ho}^{3+}$ spin disorder becomes nearly static. We see no sign of saturation of $\lambda_{\mathrm{TF}}$ down to the lowest temperature studied $(30 \mathrm{mK})$, so we infer that there may be a small dynamic component to the local field at low temperatures, as observed in longitudinal field studies on $\mathrm{LiY}_{0.955} \mathrm{Ho}_{0.045} \mathrm{~F}_{4}$ [11]. We confirmed that $\mathrm{Ho}^{3+}$ contributes to the depolarization only below $20 \mathrm{~K}$ by observing that the depolarization rates at $T=50 \mathrm{~K}$ for samples with higher Ho concentrations $(0.005 \leq x \leq 0.1)$ are identical to the results for $x=0.002$, while at low $T, \lambda_{\mathrm{TF}}$ increases with $x$.

The onset of quasistatic spin disorder of the $\mathrm{Ho}^{3+}$ ions below $20 \mathrm{~K}$ is also evident in the temperature dependence of the exponent $\beta$. A concentrated system of randomly oriented nuclear dipoles is expected to have an exponent of 2 , while for a dilute system of paramagnetic spins, the exponent is 1 for quasistatic moments and 0.5 for fluctuating moments [12]. The observed exponent is roughly 1.5 at high temperatures $(T>20 \mathrm{~K})$, reaches a minimum of 1.1 near $5 \mathrm{~K}$, and then increases to a value near 1.2 at low temperatures. The minimum in the exponent at $5 \mathrm{~K}$ indicates that the fluctuation rate is now comparable to the muon precession rate. At lower temperatures, the Ho fluc- tuations become quasistatic, and the exponent increases once again.

To probe the spin dynamics in the vicinity of the ALCs, we measured the magnetic field dependence of $\lambda_{\mathrm{TF}}$ at temperatures $T \leq 3 \mathrm{~K}$ over the range $18 \mathrm{mT} \leq H \leq$ $80 \mathrm{mT}$. We once again fit the depolarization to a stretched exponential function, with the exponent increasing slightly from $1.16 \pm 0.02$ at $18 \mathrm{mT}$ to $1.26 \pm 0.02$ at $80 \mathrm{mT}$. Data taken at $1.8 \mathrm{~K}$ are shown in Fig. 3. The most remarkable feature of the curve is the presence of sharp minima in $\lambda_{\mathrm{TF}}$ at 23,46 , and $69 \mathrm{mT}$, the field values at which the first three ALCs occur. Moreover, it is readily seen that the reductions are quite large for $n=1$ and 3 , while the reduction for $n=$ 2 is barely discernible. These minima are produced by the onset of new channels for $\mathrm{Ho}^{3+}$ spin fluctuations which are much faster than the muon precession rates $(<10 \mathrm{MHz})$, thereby reducing the (time-averaged) local magnetic disorder and decreasing $\lambda_{\mathrm{TF}}$. In general, below $4 \mathrm{~K}$, the spin disorder associated with the $\mathrm{Ho}^{3+}$ dipolar field is primarily quasistatic. However, at the resonance fields $H_{n}=$ $23 n \mathrm{mT}$, energy levels are nearly degenerate, and the $\mathrm{Ho}^{3+}$ will tunnel between the coupled energy levels with a frequency determined by the tunnel splitting: we have a new channel for enhanced spin fluctuation rates at low temperatures due to mixing between the Ho hyperfine-split states. At $n=1$ and 3, the largest tunnel splittings are predicted to be roughly $25 \mathrm{mK}$, corresponding to a characteristic frequency of $400 \mathrm{MHz}$. These fluctuations, about 100 times faster than muon precession rate, will effectively narrow the field distribution and produce a smaller spread in the time-average local field at the muon site and a decrease of $\lambda_{\mathrm{TF}}$. At $n=2$, the predicted splitting, and therefore the tunneling frequency, is much smaller, and so the reduction in $\lambda_{\mathrm{TF}}$ is also smaller.

The temperature dependence of $\lambda_{\mathrm{TF}}$ in the vicinity of the $n=1$ ALC (Fig. 4) is in qualitative agreement with the above mechanism. The sharp minimum at $23 \mathrm{mT}$ is approximately constant in size and width for temperatures between 3 and $0.9 \mathrm{~K}$. However, at $0.5 \mathrm{~K}$, the minimum becomes weaker and is no longer discernible at $30 \mathrm{mK}$.

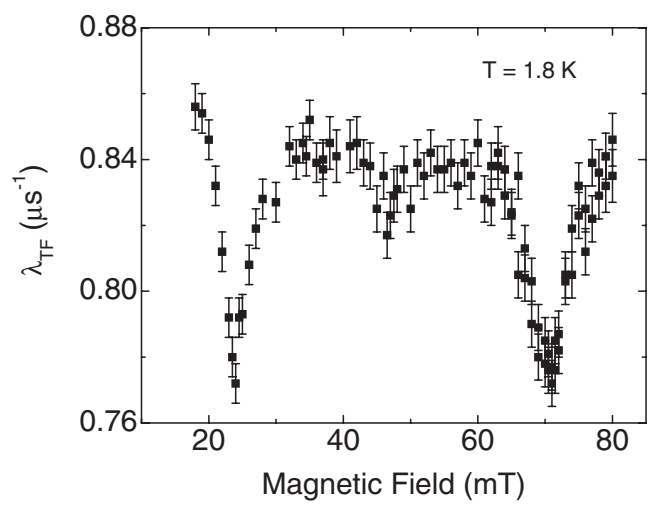

FIG. 3. Magnetic field variation of transverse field depolarization rate versus applied field at $T=1.8 \mathrm{~K}$. 


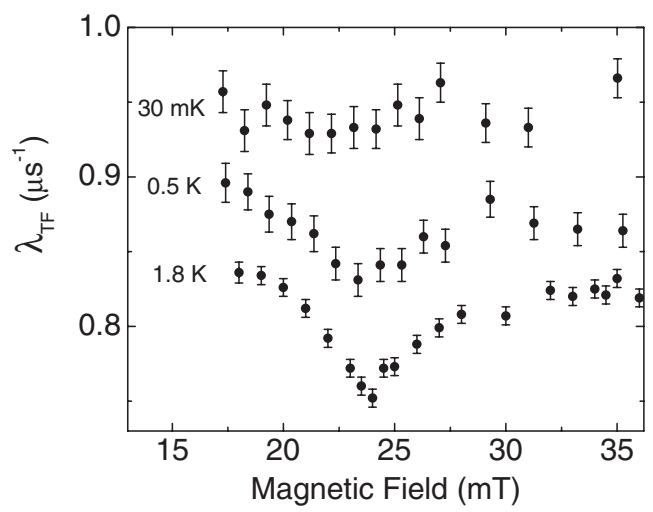

FIG. 4. Magnetic field variation of the transverse field depolarization rate $\lambda_{\mathrm{TF}}$ in the vicinity of the $n=1$ avoided level crossings at several temperatures. The curves are offset for clarity.

From Fig. 1, we see that at $23 \mathrm{mT}(n=1)$, the ALC with strong mixing is roughly $600 \mathrm{mK}$ above the lowest energy level. Fast resonant tunneling will occur for those $\mathrm{Ho}^{3+}$ ions which populate these two (nearly) crossing levels. Below $600 \mathrm{mK}$, the thermal population of these states will be reduced, and so the sharp minimum in $\lambda_{\mathrm{TF}}$ is expected to disappear, as observed.

Finally, we comment on the possible influence of the F- $\mu$-F nuclear dipolar interactions on our observations. In transverse field, the isolated F- $\mu$-F system shows a central line at the muon Larmor frequency and two sidebands with splitting of order $f_{D}$. These are just observable in the Fourier transform of high temperature data, but become indistinguishable from the central peak at low temperatures where broadening is increased. Monte Carlo simulations [10] show that in transverse fields the effect of additional interactions (electronic or nuclear) is to broaden all three lines equally, and the effective $f_{D}$ does not vary significantly. The fitted relaxation function covers all three lines, so $\lambda_{\mathrm{TF}}$ will have a constant contribution from the F- $\mu$-F splitting. Moreover, at $1.8 \mathrm{~K}$, the dc magnetization is a smooth function of magnetic field, so no abrupt changes in the static magnetic environment occur at ALCs. Our fundamental conclusion, that the change in $\lambda_{\mathrm{TF}}$ at ALCs occurs due to a change in the spin dynamics of the $\mathrm{Ho}^{3+}$, remains unchanged. Nonetheless, it would be interesting to determine the exact role of $\mathrm{F}-\mu$-F oscillations by repeating these measurements with the muon decoupled from the fluorine ions, e.g., by applying an appropriately tuned rf field.

It is remarkable that a simple electro-nuclear crystal field calculation allows us to accurately predict not only the location of level crossings, but also the degree of state mixing. This is possible because of the high crystalline quality and the excellent knowledge of the crystal field and hyperfine parameters. In this sense, the rare-earth singleion systems have a great advantage over single-molecule magnet systems, for which the local magnetic environment can be very complicated and can have a high degree of disorder. This allows for the precise characterization, and ultimately tailoring, of the magnetic properties for applications in, for example, quantum computing or information storage. Indeed, a related rare-earth-based system, $\mathrm{Er}^{3+}: \mathrm{CaWO}_{4}$, has already been demonstrated [13] to be suitable for use as a quantum qubit.

In conclusion, we have shown that transverse field $\mu^{+} \mathrm{SR}$ is a highly effective probe of the spin dynamics at avoided level crossings of the $\mathrm{Ho}^{3+}$ ion doped into $\mathrm{LiYF}_{4}$. Below $20 \mathrm{~K}$, the muon depolarization rate increases rapidly at the onset of quasistatic spin disorder associated with the $\mathrm{Ho}^{3+}$ ions. However, at avoided level crossings, this depolarization is circumvented by fast $\mathrm{Ho}^{3+}$ fluctuations resulting from quantum mixing of the states. The relative sizes and temperature dependence of the observed reductions in depolarization rates agree with the degree of level mixing predicted by selection rules resulting from perturbation theory based on a coupling of the ground state and excited electronic levels via transverse hyperfine interactions.

The authors would like to thank F. Borsa, P. Carretta, R. Kiefl, and members of the Avoided Level Crossing Group at PSI for helpful discussions, and J. Klatsky for assistance with some of the data analysis. This work was supported by the Petroleum Research Fund of the American Chemical Society, National Science Foundation Grant No. DMR-0710525, INTAS 03-51-4953, and the QUEMOLNA and MAGMANET European-NMP programs. Experiments were performed at the Swiss Muon Source, Paul Scherrer Institute, Villigen, Switzerland.

[1] F. Borsa, A. Lascialfari, and Y. Furukawa in Novel NMR andEPR Techniques, edited by J. Dolinsek, M. Vilfan, and S. Zumer (Springer, Berlin, 2006).

[2] L. Thomas et al., Nature (London) 383, 145 (1996); J. R. Friedman, M. P. Sarachik, J. Tejada, and R. Ziolo, Phys. Rev. Lett. 76, 3830 (1996).

[3] T. Lancaster et al., J. Phys. Condens. Matter 16, S4563 (2004).

[4] R. Giraud et al., Phys. Rev. Lett. 87, 057203 (2001).

[5] M. J. Graf et al., Phys. Rev. B 73, 024403 (2006).

[6] G. S. Shakurov et al., Appl. Magn. Reson. 28, 251 (2005).

[7] M.J. Graf et al., Physica (Amsterdam) 374B-375B, 9 (2006).

[8] See for example A. Amato, Rev. Mod. Phys. 69, 1119 (1997).

[9] J. H. Brewer et al., Phys. Rev. B 33, 7813 (1986).

[10] J.S. Lord (unpublished).

[11] J. Rodriguez et al., Physica (Amsterdam) 374B-375B, 13 (2006).

[12] Y. J. Uemura, in Muon Science: Muons in Physics, Chemistry and Materials, edited by S. L. Lee, S. H. Kilcoyne, and R. Cywinski (IOP Publishing, Bristol, 1999).

[13] S. Bertaina et al., Nature Nanotechnology 2, 39 (2007). 